\title{
QUALITY IMPROVEMENT The frailest of the frail? Addressing the palliative care needs of frail older patients
}

\author{
Authors: Raymond Hyatt, ${ }^{\mathrm{A}}$ John Dean, ${ }^{\mathrm{B}}$ Marie O'Niell, ${ }^{\mathrm{C}}$ Nikesh Patel, ${ }^{\mathrm{D}}$ Mustafa Abu-Rabia ${ }^{\mathrm{E}}$ and Joanne Taylor ${ }^{\mathrm{F}}$
}

\begin{abstract}
Managing patients with frailty is an increasing element of acute hospital care, constituting around $40 \%$ of acute hospital inpatients. Up to half of these patients may be approaching the end of their life, and many also have repeated hospital admissions. We therefore undertook a quality improvement project to help us identify the prevalence of frail older inpatients approaching end of life and to better identify these patients in advance. We also developed and evaluated a proforma to assist in initiating an advance care planning conversation with the patient and/or family. Fifty percent of our frail older inpatients were assessed as approaching end of life. Factors identifying older inpatients as more likely to die during the hospital admission included residence in a care home, two or more hospital admissions over the preceding 12 months, and a diagnosis of dementia for more than 3 years. A novel goals and priorities of care (GPOC) document was found to support and guide palliative care conversations. Acute hospital care could be organised more effectively to recognise the potential need for palliative care in frail older patients. Identifying those at higher risk and using structured interviews and documentation is helpful, ultimately resulting in more appropriate care. Well-developed communication skills are needed for these complex care planning conversations.
\end{abstract}

KEYWORDS: frail older patients, last year of life; palliative care conversations

\section{Introduction}

This work was undertaken as part of the Royal College of Physicians (RCP) Future Hospitals Programme (FHP) from October 2014 to November $2017^{1}$ at East Lancashire Hospitals

Authors: ${ }^{A}$ Consultant geriatrician, East Lancashire Hospitals NHS Trust, Blackburn, Lancashire, UK; ${ }^{\text {B }}$ deputy medical director and consultant physician, East Lancashire Hospitals NHS Trust, Blackburn, Lancashire, UK; ' Ward Manager, East Lancashire Hospitals NHS Trust, Blackburn, Lancashire, UK; ${ }^{\text {D }}$ specialty trainee in geriatric medicine, Salford Royal NHS Foundation Trust, Salford, UK; ${ }^{{ }}$specialty trainee in geriatric medicine, Bolton NHS Foundation Trust, Bolton, UK; ${ }^{{ }}$specialty trainee in geriatric medicine, Central Manchester University Hospitals NHS Foundation Trust, Manchester, UK
NHS Trust as a phase 1 FHP development site. Phase 1 sites focussed on improving care for frail older people. Our aim was to improve the design and delivery of a frailty pathway, spanning both hospital and community settings, in line with the principles of the Future Hospital Commission. ${ }^{2}$ This report comprises one component of our FHP work; other elements are reported elsewhere. ${ }^{1,3,4}$

Frailty, by definition, identifies those at risk of adverse outcomes, including death, ${ }^{5}$ and previous studies have demonstrated a high mortality in frail older medical inpatients. ${ }^{6,7}$ The General Medical Council draws a distinction between patients likely to die imminently (hours or days) and those expected to die within the next 12 months, which it describes as approaching the end of life. ${ }^{8}$ This approach is incorporated into palliative care practice in the form of the Gold Standards Framework and the surprise question ('Would I be surprised if this patient died in the next twelve months?' ${ }^{9}$ However, frail older patients are generally underrepresented in primary care palliative care registers. ${ }^{10}$

The recognition of approaching end of life, particularly in frail older patients, is not straightforward, given the often unpredictable course of the functional decline associated with frailty. ${ }^{11}$ As a consequence, the palliative care needs of these patients often go unrecognised and conversations about possible outcome and appropriate treatment with patients, families and carers may be limited or may not take place.

Comprehensive Geriatric Assessment (CGA) is the gold standard methodology to assess the needs of frail older patients presenting to acute care. ${ }^{12}$ However, in practice, when frail older patients present there is often a focus on pathways for treatments of single conditions (eg sepsis) or single organs, rather than assessment of holistic needs, including the consideration of whether palliative care may be appropriate. ${ }^{13}$ This process may be repeated uncritically, resulting in multiple admissions as patients approach the end of life. This may be unhelpful for the patient, and not what they and/or their family would have wished. This pattern of care had been observed by the authors and forms the rationale for this work.

This project was undertaken in East Lancashire Hospitals NHS Trust, predominantly in Royal Blackburn Hospital, a large acute hospital serving a population of more than 500,000 . It focuses on the prevalence of frail older patients approaching end of life, the potential for their prospective identification and the development of an effective proforma to support the initiation of palliative care conversations with patients and/or their families. 


\section{Methods}

Surveys of prevalence of frailty and approaching end of life

In order to contextualise this work, and for operational purposes, we undertook a point prevalence survey of inpatients on one weekday in our hospitals. For the purposes of this survey, being aged over 80 years old and an inpatient within the medical division on the day of the survey was accepted as a proxy for frailty. The limitations of this definition are acknowledged. Data was acquired using hospital episode statistics.

The prevalence of frail older patients assessed as likely to be in the last 12 months of life was then identified as part of a separate, more detailed survey of the clinical characteristics, process and outcome of care, of 50 randomly selected frail older inpatients in acute beds at Royal Blackburn Hospital within the medical division. This work was undertaken over a one week period by a multidisciplinary team (MDT) skilled in the assessment of frail older patients, using a structured questionnaire (Frail Older Patient Admission Questionnaire: FOPAQ). The FOPAQ questionnaire was tested and adapted by the MDT using an iterative process, and was accepted in its final form at its 10th iteration. Other details of the questionnaire have been reported elsewhere. ${ }^{4}$

In order to meet a definition of frail for FOPAQ, the patient was over 80 years old, and admitted with one of confusion, falls, or poor mobility, or from a care home. This pragmatic definition of frailty has been used elsewhere. ${ }^{14}$ The first 50 medical inpatients to meet this definition of frailty over the one week period of the survey were selected for the FOPAQ survey. The FOPAQ questionnaire also included the 'surprise question', ie 'would I be surprised if this patient died within the next 12 months'? ${ }^{15}$

\section{Retrospective casenote audit}

We then undertook a retrospective case-note review of clinical markers which, based partly on review of the relevant literature, ${ }^{16,17}$ and partly on clinical observation, we judged might best identify older patients approaching end of life. These are shown in Box 1.

We retrospectively reviewed 80 case notes of patients over 80 years of age admitted consecutively to the division of medicine within the preceding 6 months, comparing 40 case notes of patients who had died in hospital, with 40 case notes of patients who had survived to discharge. The first 40 available case notes of

Box 1. Characteristics explored in retrospective case note review

$>$ Numbers of prior admissions in the last 12 months

$>$ Location prior to admission (own home or care home ie residential or nursing home)

> Clinical Frailty Scale (CFS) score, ${ }^{15}$ - scored retrospectively from Generic Admission Document

Presence of diagnosis of Dementia

$>$ Time from diagnosis of Dementia to admission

$>$ Co Morbidities

Death anticipated during admission?

> Evidence of DNACPR, Advance Care Plan and/or Goals and Priorities of Care Discussion eligible patients for each group selected by the audit department were reviewed. There was no formal process of randomisation of the case notes reviewed.

\section{Evaluation of goals and priorities of care (GPOC) proforma}

This element of the project took place in acute GIM/medicine for older people wards. The form was used by senior clinicians including the first author, senior medical trainees, and the ward manager. The patient group selected were frail older patients identified using the criteria previously described, who were assessed by a senior clinician and the ward MDT, on clinical grounds, as appropriate for an advance care planning (ACP) discussion. These took place with the senior clinician and family and/or patient.

The aim of the GPOC proforma was to better structure palliative care conversations in the setting of frailty, and better identify the goals and priorities of care for frail older patients and their families. It incorporated issues specifically relevant to frail older patients and their families; such as assisted nutrition and hydration, intravenous therapy including antibiotics, and readmission to hospital. The GPOC proforma was designed, tested and adapted through a series of PDSA cycles. Initial drafts were based on treatment escalation plans (TEPS) which had been used in other health economies. ${ }^{18,19}$

The iterative process involved feedback from key stakeholders, including the Trust end of life steering group, palliative care physicians, and those testing the use of the form. The final draft of the proforma was adopted after its 10th iteration (see online Appendix).

The proforma also contained a section for completion by the professional leading the conversation, including space to record the duration of the conversation, a self-assessment of the quality of the conversation (/10), a similar question on quality of conversation for any other professional in attendance, and an assessment of how challenging it felt to undertake (/10).

\section{Results}

\section{Prevalence of frailty and those approaching end of life}

Our point prevalence survey of all medical inpatients in the Trust, including community hospitals, on a single day demonstrated that 330 of 829 inpatients were over 80 years of age: a prevalence of $39.8 \%$.

The FOPAQ questionnaire survey then demonstrated that of a sample of 50 frail medical inpatients over the age of 80,25 (50\%) were assessed as likely to die within the next 12 months and were therefore approaching end of life. ${ }^{8}$

\section{Retrospective case note review}

The results of the retrospective case note review of the 2 cohorts of patients is shown in Table 1. Patents who died during the admission were more likely to have had two or more hospital admissions in the preceeding 12 months, a diagnosis of dementia for more than 3 years, and have been living in a care home, compared to those who survived the admission.

\section{GPOC proforma evaluation}

Forty frail patients and/or their families had an ACP conversation using the GPOC proforma. 14 conversations took place on an acute 


\begin{tabular}{|c|c|c|}
\hline & $\begin{array}{l}\text { Patients over } \\
80 \text { died during } \\
\text { admission } \\
\text { episode } \\
\text { ( } 40 \text { patients) }\end{array}$ & $\begin{array}{l}\text { Patients over } \\
80 \text { survived } \\
\text { to discharge } \\
\text { ( } 40 \text { patients) }\end{array}$ \\
\hline Male & 16 & 12 \\
\hline Female & 24 & 28 \\
\hline Age (years) & $\begin{array}{l}\text { Median } 88.2 ; \\
\text { range } 82-98\end{array}$ & $\begin{array}{l}\text { Median } 86.5 \\
\text { range } 80-95\end{array}$ \\
\hline $\begin{array}{l}\text { Two or more admissions in } \\
\text { last } 12 \text { months (number) }\end{array}$ & 22 & 9 \\
\hline Place prior to admission & $\begin{array}{l}\text { Own home 23; } \\
\text { care home } 17\end{array}$ & $\begin{array}{l}\text { Own home 29; } \\
\text { care home } 11\end{array}$ \\
\hline $\begin{array}{l}\text { Diagnosis of dementia } \\
\text { (number) }\end{array}$ & 12 & 9 \\
\hline $\begin{array}{l}\text { Diagnosis of dementia } \\
>3 y \text { (number) }\end{array}$ & 8 & 3 \\
\hline Clinical frailty scale & $\begin{array}{l}\text { Median 6; } \\
\text { range 3-8 }\end{array}$ & $\begin{array}{l}\text { Median 5; } \\
\text { range } 3-8\end{array}$ \\
\hline$>6$ comorbidities (number) & 36 & 36 \\
\hline DNA CPR in place (number) & 29 & 6 \\
\hline $\begin{array}{l}\text { Advance care planning } \\
\text { conversation (number) }\end{array}$ & 13 & 16 \\
\hline
\end{tabular}

medicine for older people ward, 25 on an acute orthogeriatric ward, and one on a medical admissions unit. Outcomes of the conversations and characteristics of the patients are shown in Table 2.

The following elements of the GPOC form and process were noted and adjusted during the PDSA cycles:

$>$ Initial drafts of the form included a number of specific potential medical interventions (eg inotropic support). Covering these individually was found to be unnecessary.

> Time to complete the conversation was noted to sometimes take longer than initially anticipated.

$>$ Advanced communication skills were also noted to be important; in several cases the conversation was not expected by the patient's family; in one conversation the lead clinician felt they hadn't allowed sufficient opportunity for the patient's family to identify their concerns.

The following were noted to be helpful in advanced care planning:

> Specific discussion around CPR; escalation to level 2/3 care; artificial feeding; IV antibiotics; IV fluids; readmission.

> The conversation could be led and the form completed by different healthcare professionals eg by consultant, senior trainee/middle grade doctor, and senior non-medical member of the MDT (ward manager).

$>$ Structured conversation was generally well received by patients and families.
Table 2. GPOC conversation outcomes in $\mathbf{4 0}$

\section{consecutive patients}

\section{Characteristics of patients}

Age (years)

Median 87; range 65-97

Clinical Frailty Scale

Median 8; range 6-9

Number of comorbidities

Has mental capacity - Yes/No

Median 6; range 2-14

$10 / 30$

\section{Characteristics of discussion}

Duration of discussion (min)

Median 30; range 5-75

Discussion involved patient - Yes/No

(if no, family only)

Discussion led by

$5 / 35$

Consultant: 25

Doctor in training: 14

Ward manager: 1

Outcome of conversation

Care focussed on cure

0

Care focussed on disease control

Care focussed on comfort

CPR appropriate/inappropriate

Readmission appropriate/

$22 / 18$

inappropriate

Artificial feeding/ IV or SC fluids/

$30 / 10$

further oral or IV antibiotics

specifically excluded - Yes/No

\section{Clinician assessment of conversation (available for 26/40)}

Quality of discussion - self-rated by lead of conversation (/10):

Quality of discussion - rated by observer (/10)

How challenging - self-rated by lead of conversation (/10)

Median 8; range 6-9

Median 8; range 7-10

Median 5; range 1-10

The following is a representative sample of feedback in free text obtained about the GPOC-facilitated conversations from the lead/ user:

$>$ '....Already agreed for palliative care if treatment is not successful in the next 12-48 hours....'

> '....Overall went well, agreed: DNACPR, not for feeding, not for readmission, in effect for palliative management....'

> '....Consultation went well, challenging, family was not expecting an emergency....

> '....Difficult (bedside, family came in half way though), patient not prepared for discussion, not broached by Oncology (why?)....'

> '....Pts daughter had been apparently aggressive with trainees, so was apprehensive, but went well overall, family seemed to want more info and were satisfied with explanation...'

> '....the start of a conversation nobody else had broached!...'

A word cloud from the narrative in the GPOC form is shown in Fig 1. 


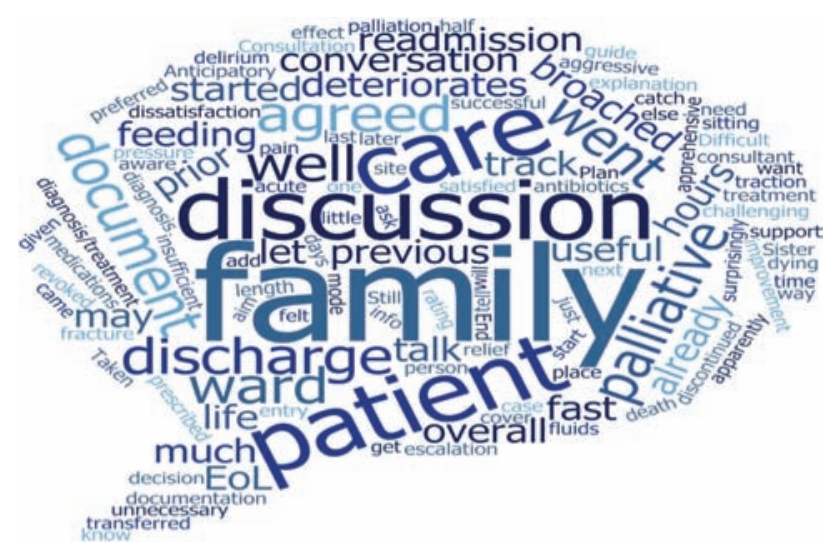

Fig 1. A word cloud from the narrative in the GPOC form.

\section{Discussion}

This was a pragmatic quality improvement project, undertaken by experienced clinicians in a typical acute hospital in England, addressing a commonly encountered patient group. We found that of the $40 \%$ of inpatients who were frail older people, $50 \%$ were likely to be in their last year of life. Frail older people who died during a hospital admission were more likely to have had multiple hospital admissions, long standing dementia and live in a care home. Having a structured approach to care planning conversations with these patients and their families leads to decisions that might reduce inappropriate hospitalisation and intervention. While there are limitations to the methodology, we believe the results and conclusions are generally applicable, and can guide both clinical practice, and further quality improvement and research.

Limitations of the work include pragmatic definitions of frailty, retrospective case note review rather than real time clinical assessment of patients admitted with frailty syndromes, a relatively small sample size for the comparative retrospective review, and lack of a randomised sampling method for case selection. The patients who died in the cohort review died during the episode admission, and those who survived to discharge may have died during subsequent admissions or at home. The GPOC was performed predominantly in two wards by a limited number of clinicians.

The finding that $40 \%$ of inpatients were over 80 years of age is consistent with other published data, demonstrating that a substantial and growing proportion of inpatient beds are occupied by patients over 65 years. ${ }^{20}$ Similarly the finding that $50 \%$ of frail older inpatients were assessed as approaching end of life is consistent with other larger studies, 8,9 and in the authors' opinion is not commonly reflected in clinical practice.

The widely used Clinical Frailty Scale (CFS) has two explicit categories for patients approaching end of life. ${ }^{21}$ Use of this scale is now becoming more common, and while this can guide our clinical decision making, this was not a clear discriminator in our work. In the retrospective case note review CFS scores were unexpectedly low despite the fact that patients in both cohorts had multiple comorbidities. This may reflect the difficulty of scoring the CFS retrospectively and the possibility that information regarding premorbid functional status may not have been accurately recorded on admission.
The majority of frail older patients who died had had a DNACPR form completed; as this was a retrospective study, the time that this was completed may have been shortly prior to death or prior to admission. A significant minority did not have DNACPR or advance care planning in place despite the fact that this was their third (or more) admission within 12 months. Indeed, relatively few patients in either cohort had had a documented ACP conversation recorded in the case-notes, despite mechanisms existing locally for these to be transferred with patients on admission from their residence.

A high prevalence of frailty and dementia in care home residents is well established and these patients are frequently admitted acutely when approaching end of life. ${ }^{20,21}$ Increased mortality in older inpatients with dementia is unsurprising as Alzheimer's disease and other dementias are now the leading cause of death in the UK. ${ }^{22}$ The median survival of older patients diagnosed with dementia ranges from 6.7-1.9 years, falling steeply with age at diagnosis. ${ }^{23}$ Multiple hospital admissions are also established as being characteristic in patients approaching end of life; ${ }^{15}$ however, there again appears limited awareness of the potential significance of this in usual clinical practice. ${ }^{24}$

The use of a structured template to guide complex care planning discussions was found to be helpful for staff and families. Prior to this no formal document or guide other than DNACPR was used. It should be noted that the majority of discussions were with families as most of these patients did not have capacity for the discussion and decisions. These were often difficult and complex discussions. This required skills that are acquired by experienced practitioners, and we recommend that in the future they become core skills, formally developed for physicians and other clinical team members. The time required for a conversation using this approach can be considerable, with two conversations lasting more than one hour. This can be difficult to accommodate in the acute clinical setting; however, there is an overall benefit within the wider service, as it reduces the use of resources in the longer term.

The need to develop more inclusive discussions for patients approaching the end of life, rather than the binary nature of DNACPR, is recognised through the development of the ReSPECT approach, led by the Resuscitation Council. ${ }^{25}$ This is also likely to have potential use for frail older patients and/or their families. However, the level of detailed decision making and documentation included in the GPOC discussions was more than is included in the ReSPECT proformas. We believe that the two approaches are complementary. Existing approaches, including provision of palliative care input to care homes where appropriate ${ }^{26}$ and the consideration of palliative care needs of patients diagnosed with dementia, may also help address this frequently unmet need. ${ }^{27}$

Significant decisions regarding less intensive treatment, or the desire to avoid hospital readmission, were made using the GPOC process; half of patients with families requested not to be readmitted if their condition deteriorated after discharge and a quarter of patients with families agreed to less invasive treatments at ward level. This has significant implications for future care demand, both in hospital and in the community, and requires a whole-system approach. Communication of the outcome of GPOC in the discharge summaries was included, with the request to the primary care team to continue advance care planning discussions.

The primary goal of geriatric medicine has been and remains the identification and treatment of reversible disease in older people. Comprehensive Geriatric Assessment (CGA) is the evidence based tool to support this process. ${ }^{12}$ The identification of frail older 
patients approaching the end of their life should be via timely CGA delivered in the appropriate setting, followed by advance care planning if appropriate. This approach is likely to improve care, may avoid unsought and unhelpful hospital admissions, and is consistent with national guidance. ${ }^{28}$

The simple clinical markers described in this paper may help identify a cohort of frail older patients approaching end of life. Using instruments such as the GPOC proforma might facilitate the start of a conversation about the potential for palliative care where appropriate, leading to further ACP and ReSPECT documentation.

Further work in this area will require a system-wide approach; better communication between primary and secondary care, greater input of specialist palliative care services for the care of frail older patients and investment in the development of advanced communication within the workforce will facilitate this. Involvement of other healthcare disciplines, social care professionals and the voluntary sector are needed. Emergent Accountable Care Systems in England are well placed to do this, focusing on managing population need rather than activity.

The report from the Parliamentary and Health Service Ombudsman (PHSO) recounts the experience of frail older patients dying in hospital and observes that '....the reasonable expectation that an older person or their family may have of dignified, painfree end of life care, in clean surroundings in hospital, is not being fulfilled.... ${ }^{29}$ There is a critical need for feedback from patients and families to inform service design for these scenarios.

In order to achieve this, frail older patients need to have had robust CGA and their palliative care needs recognised and actioned appropriately. We hope the measures outlined in this paper may contribute to achieving this hitherto largely unfulfilled aim.

\section{References}

1 Royal College of Physicians. Delivering the future hospital. RCP, 2017. Available online at www.rcplondon.ac.uk/projects/outputs/ future-hospital-programme-delivering-future-hospital

2 Royal College of Physicians. Future hospital: caring for medical patients. RCP, 2013. Available online at www.rcplondon.ac.uk/projects/ outputs/future-hospital-commission [Accessed 11 January 2018].

3 Dean J. Improving acute care for frail older people in East Lancashire - Future Hospital. Poster presented at the International Forum for Quality and Safety in Healthcare. Gothenburg, 2016.

4 Hyatt R, Taylor JK, Robertson $M$ et al. Measuring the process of care of frail older patients in hospital: the frail older patient admission questionnaire (FOPAQ). Age and Ageing 2017;46:i1-i22.

5 Moorhouse P, Rockwood K. Frailty and its quantitative clinical evaluation. J R Coll Physicians Edinb 2012;42:333-40.

6 Eeles $\mathrm{E}$, White S, O'Mahony S et al. The impact of frailty and delirium on mortality in older inpatients. Age and Ageing 2005;34:432-4.

7 Clark D, Armstrong M, Allan A et al. Imminence of death among hospital inpatients: prevalent cohort study. Palliat Med 2014;28:474-9.

8 General Medical Council. Treatment and care towards the end of life: good practice in decision making. GMC, 2010.

9 Gold standard Framework. The GSF prognostic indicator guidance. GSF, 2011. Available online at www.goldstandardsframework.org. uk/cd-content/uploads/files/General \% 20Files/Prognostic \% 20 Indicator \% 20Guidance \% 200ctober \% 202011.pdf [Accessed 11 January 2018].
10 Eynon T, Lakhani M, Baker R. Never the right time: advance care planning with frail and older people. Br J Gen Pract 2013;63:51112.

11 Lunney J, Lynn J, Foley D et al. Patterns of functional decline at the end of life. JAMA 2003;289:2387.

12 Ellis G, Gardner M, Tsiachristas A et al. Comprehensive geriatric assessment for older adults admitted to hospital. Cochrane Database Systematic Reviews 2017;9:CD006211.

13 National Mortality Case Record Review Programme. West of England Academic Health Science Network (WEAHSN) mortality case study. RCP, 2017. Available online at www.rcplondon.ac.uk/ projects/outputs/west-england-academic-health-science-networkweahsn-mortality-case-study.

14 Oo M, Tencheva A, Khalid N et al. Assessing frailty in the acute medical admission of elderly patients. J $R$ Coll Physicians Edinb 2013:43:301-8.

15 Royal College of General Practitioners. The GSF prognostic indicator guidance. RCGP, 2011.

16 Lyons $\mathrm{P}$, Verne J. Pattern of hospital admission in the last year of life. BMJ Support Palliat Care 2011;1:81-2.

17 Smith P, Sherlaw-Johnson C, Ariti C, Bardsley M. Focus on: hospital admissions from care homes. Health Foundation, 2015. Available online at www.health.org.uk/sites/health/files/QualityWatch_ FocusOnHospitalAdmissionsFromCareHomes.pdf [Accessed 11 January 2018].

18 Cambridge University Hospitals Foundation Trust. Universal Form of Treatment Options (UFTO). CUH, 2014. www.cuh.nhs.uk/addenbrookes-hospital/for-patients/patient-information-and-consentforms/universal-form-treatment-options-ufto

19 Karakusevic C. Devon treatment escalation plan and resuscitation decision record (TEP). Macmillan, 2013. www.macmillan.org.uk/ documents/aboutus/health_professionals/primarycare/showcasing/2013/treatment-escalation-and-resuscitation-devon.pdf

20 Oliver D, Foot C, Humphries R. Making our health and care systems fit for an ageing population. Kings Fund, 2014.

21 Rockwood K, Song X, MacKnight C et al. A global clinical measure of fitness and frailty in elderly people. CMAJ 2005;173:489-95.

22 Office for National Statistics. Deaths registered in England and Wales. ONS, 2015.

23. Rait G, Walters K, Bottomley $C$ et al. Survival of people with clinical diagnosis of dementia in primary care: cohort study. BMJ 2010;341:c3584.

24 Age UK. End of Life Evidence Review. Age UK; 2013

25 Fritz Z, Slowther A, Perkins G. Resuscitation policy should focus on the patient, not the decision. BMJ 2017;356:j813.

26 The National Council for Palliative Care. Introductory guide to end of life care in care homes: NHS End of Life Care Programme. NCPC, 2006.

27 Lloyd-Williams M, Mogan C, Dening K. Identifying palliative care needs in people with dementia; Curr Opin Support Palliat Care;11:328-3.

28 Cooke M, Oliver D, Burns A. Quality care for older people with urgent and emergency care needs ('Silver Book 2012'). British Geriatric Society, 2012. Available online at www.bgs.org.uk/campaigns/silverb/ silver_book_complete.pdf

29 Parliamentary and Health Service Ombudsman. Care and compassion? PHSO; 2011.

Address for correspondence: Dr Raymond Hyatt, East Lancashire Hospitals, Royal Blackburn Hospital, Haslingden Road, Blackburn BB2 3HH.

Email: raymond.hyatt@elht.nhs.uk 